\title{
ESTUDO DA TRANSFERENCIA DE CALOR PROMOVIDA POR UM SISTEMA DE BICOS INJEÇÃO DE AR QUENTE SOBRE UMA SUPERFÍCIE DE ALGODÃO
}

\author{
J.W. PARRA, M. B. QUADRI \\ Universidade Federal de Santa Catarina, Departamento de Engenharia Química e Engenharia \\ de Alimentos. \\ E-mail para contato: eng.johnparra@gmail.com, m-quadri@enq.ufsc.br
}

\begin{abstract}
RESUMO - Simula-se a transferência de calor promovida por jatos de ar gerados a partir de bicos com borda hemi-toroidal direcionados perpendicularmente sobre uma superfície de algodão, considerando-se a interação fluidodinâmica entre esses jatos. Utiliza-se um modelo de escoamento turbulento do tipo $\kappa-\varepsilon$ implementado no software Comsol Multiphysics 4.3b. Adotou-se uma geometria 3D com cinco bicos injetores (uma fila com três e outra com dois) com diâmetro de $0,015 \mathrm{~m}$. A velocidade de entrada e a temperatura do ar são $2 \mathrm{~m} / \mathrm{s}$ e 453,15 K, respectivamente. Os valores testados para a razão entre a altura dos jatos e o diâmetro dos bicos $\left(\mathrm{H} / \mathrm{D}_{\mathrm{b}}\right)$ são de 2,0; 4,6 e 6,0. A superfície de algodão entra com temperatura de $293,15 \mathrm{~K}$ e velocidade entre $0,2 \mathrm{~m} / \mathrm{s}$ e $5,0 \mathrm{~m} / \mathrm{s}$. Perfis de velocidade e de temperatura, além dos números de Nusselt são presentados. Os coeficientes de transferência de calor locais chegaram ao valor máximo de 41,8 $\mathrm{W} / \mathrm{m}^{2} . \mathrm{K}$, o que é considerado adequado diante de referências da literatura afim.
\end{abstract}

\section{INTRODUÇÃO}

A transferência de calor por meio de jatos está presente em diversas aplicações industriais, tais como: têmpera e modelagem de vidro, recozimento de metais, secagem de produtos têxteis e de papel, e resfriamento das aletas das turbinas e equipamentos eletrônicos segundo Colucci e Viskanta (1996). Na literatura podemos encontrar diversos estudos numéricos sobre jatos de ar turbulentos onde se comparam diferentes tipos de modelos de turbulência com dados experimentais. Colucci e Viskanta (1996) estudaram o efeito de um bico de geometria hiperbólica sobre os coeficientes de transferência de calor locais usando uma técnica de cristais líquidos termo cromáticos para visualizar e gravar isotermas sobre uma superfície de impacto uniformemente aquecida. Eles concluem que os coeficientes de transferência de calor no local de incidência de jatos confinados são mais sensíveis ao número de Reynolds e ao espaçamento entre o bico e a placa do que aqueles para jatos não confinados. Gulati et al (2008) estudaram os efeitos da forma do bocal, o espaçamento entre o mesmo e a placa e o número de Reynolds na distribuição de transferência de calor local de um jato de ar sobre uma superfície lisa e plana. Sharif e Banerjee (2008) estudaram a transferência de calor por convecção sobre uma placa quente em movimento com velocidades entre 0 e $2 \mathrm{~m} / \mathrm{s}$, o estudo mostrou que o número de Nusselt médio aumenta consideravelmente com o aumento do número de Reynolds e diminuem com o aumento da velocidade da placa. Chattopadhyay e Saha (2003) investigaram a transferência de calor para um jato sobre uma 
placa em movimento usando o modelo de turbulência LES para um número de Reynolds de 5800 .

Neste contexto, o presente trabalho se propõe a estudar a transferência de calor promovida por um conjunto de cinco bicos que injetam ar quente perpendicularmente sobre uma camada plana de algodão que pode se deslocar longitudinalmente a uma dada velocidade. Esse sistema se relaciona ao que pode ser encontrado em um secador Rama de circulação cruzada, comumente utilizado na indústria têxtil. Procura-se determinar os números de Nusselt e os coeficientes convectivos locais de modo a avaliar a eficiência da transferência de calor neste tipo de equipamento.

\section{GEOMETRIA, MALHA E CONDIÇÕES DE FRONTEIRA}

Para a construção da geometria, adotou-se como modelo um injetor de uma Rama como se mostra na Figura 1, adaptada para uma disposição lateralmente desencontrada de cinco bicos. Os secadores Rama são equipamentos térmicos utilizados na secagem de tecidos em processo contínuo. Nas câmaras de secagem, ar aquecido flui sobre o tecido de forma perpendicular e o seca convectivamente com a troca de massa e calor (Ferraz et al., 2010).
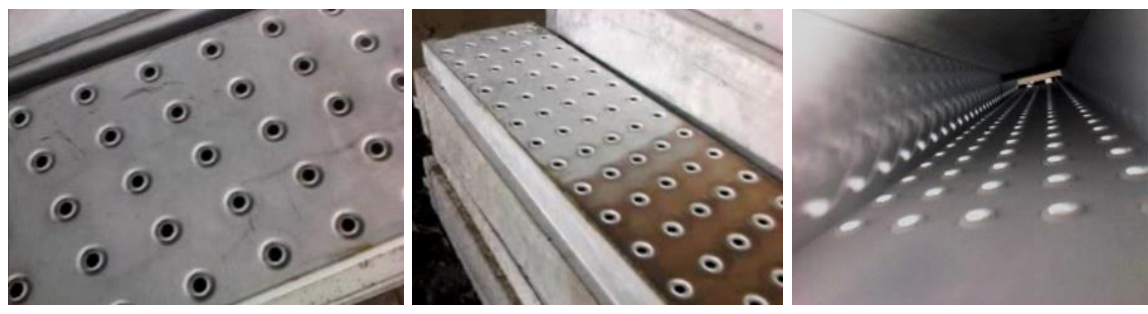

Figura 1 - Fotos de um injetor de um secador Rama.

Construiu-se uma geometria 3D composta de dois domínios: o primeiro compreende o espaço de altura $\mathrm{H}$ entre a superfície superior da placa de algodão e a parede superior onde se encontram os bicos injetores; o segundo, na região inferior, representa a camada de algodão segundo uma placa plana em movimento que entra a uma temperatura $T_{\text {placa }}$ de $293.15 \mathrm{~K}$. Os bicos injetores são em número de cinco (uma fila com três e outra com dois, desencontradas lateralmente) seccionados longitudinalmente ao meio segundo dois planos verticais laterais que são assumidos como planos de simetria para efeito dos cálculos de simulação. Ao se definir essa geometria, pretende-se economizar malha e por consequência esforço computacional. Foram utilizadas as seguintes condições de contorno: impôs-se a condição função de parede (no slip) às paredes dos bicos e à fronteira superior da geometria, estas também foram isoladas termicamente, juntamente com a parede inferior da placa plana que corresponde à camada de algodão; condições de contorno de simetria foram adotadas para as paredes laterais; e de fronteira aberta no domínio superior para os lados correspondentes à entrada e saída do algodão; para a fronteira inferior do domínio superior que coincide com a parede superior da camada de algodão foi usada a condição de parede deslizante. $\mathrm{O}$ ar entra pela fronteira superior de cada bico a uma velocidade $V_{\text {ar }}$ de $2 \mathrm{~m} / \mathrm{s}$ e temperatura $T_{\text {ar }}$ de 453,15 K. Além disso, a camada de algodão fica submetida a um movimento de translação com velocidade prescrita. 
No método de elementos finitos, empregado neste estudo, a construção da malha é um dos aspectos mais importantes para a correta convergência de uma simulação. Onde mais se tomou cuidado com a malha foi junto às paredes dos bicos e junto à placa de algodão. Para isto se fez um estudo de malha variando o tamanho e número dos elementos nessas regiões, como se mostra na Figura 2a, a fim de se ter melhores resultados nas simulações.

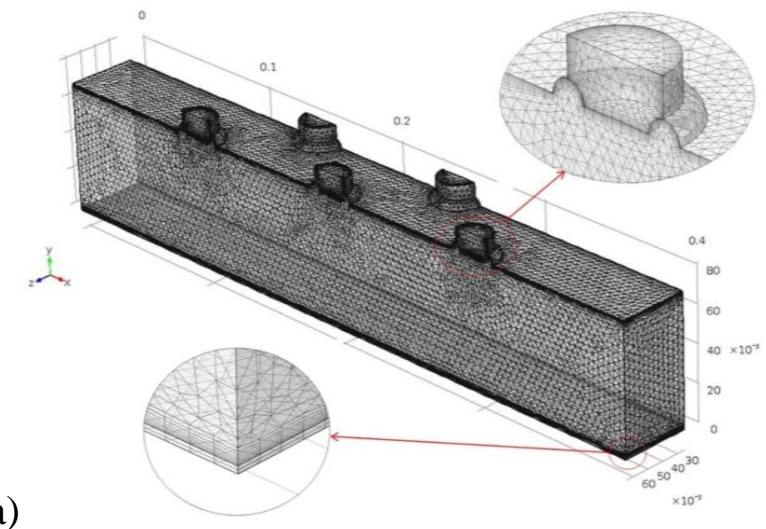

b)

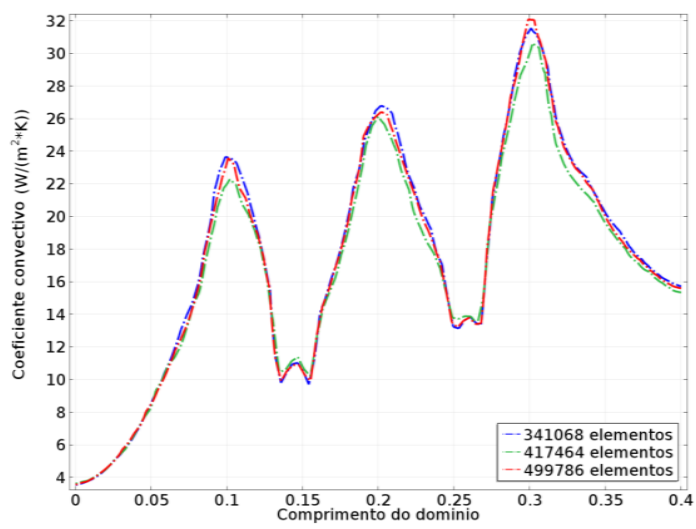

Figura 2 - (a) Malha com detalhes utilizada na discretização do domínio de cálculos. (b) Coeficientes convectivos de calor para diferentes malhas.

No estudo de malha foram usados os seguintes valores $V_{a r}=2 \mathrm{~m} / \mathrm{s}, V_{\mathrm{p}}=0,4 \mathrm{~m} / \mathrm{s} \mathrm{e} \mathrm{H} / \mathrm{D}_{\mathrm{b}}$ para três malhas com 341.068, 417.464 e 499.786 elementos. Os coeficientes convectivos de transferência de calor locais na superfície da camada de algodão, ao longo da linha definida pelos centros dos três bicos alinhados, para as três malhas testadas são apresentados na Figura 2 b. Pode-se observar que não há grande diferença nos valores, mas ao se adotar oito camadas hexaédricas para as paredes dos bicos e sobre a superfície do algodão para a malha de 417.464 elementos, notou-se uma pequena redução no valor dos coeficientes para os pontos correspondentes às regiões centrais dos bicos. Para as outras duas malhas, foram utilizadas quatro camadas hexaédricas. Julga-se que com oito camadas a estimativa dos gradientes de temperatura, necessária para o cálculo desses coeficientes, é mais precisa do que com quatro camadas. Assim, optou-se pela malha de 417.464 elementos para realizar todas as simulações subsequentes deste trabalho.

A região onde o ar é injetado corresponde a um paralelepípedo $0,4000 \mathrm{~m}$ de comprimento, $0,1300 \mathrm{~m}$ de largura e altura de $0,0700 \mathrm{~m}$. Para este modelo se adotou uma intensidade de turbulência constante de $5 \%$ e uma escala longitudinal de turbulência de $\left(0,07 * D_{H}\right)$ onde, $D_{H}$ é o diâmetro hidráulico do bico. O número total de bicos é de 5 . A forma de casca hemi-toroidal do bico objetiva distribuir o jato de ar de forma mais eficiente sobre a superfície de algodão. Os bicos têm diâmetro $D_{b}=0,0150 \mathrm{~m}$ e altura $A_{b}=0,005 \mathrm{~m}$. Para facilitar a convergência do modelo, estenderam-se cilindros sobre os bicos com diâmetro $D_{\text {cil }}=$ $0,0150 \mathrm{~m}$ e uma altura $\mathrm{A}_{\text {cil }}=0,0075 \mathrm{~m}$. A altura inicial entre os bicos e a placa de algodão é $\mathrm{H}=0,0700 \mathrm{~m}$. Para a camada de algodão, considerou-se uma placa plana de 0,4000 $\mathrm{m}$ de comprimento, $0,1300 \mathrm{~m}$ de largura e $0,0015 \mathrm{~m}$ de altura, com temperatura de entrada de $\mathrm{T}_{\text {placa }}$ $=293,15 \mathrm{~K}$. As seguintes propriedades físicas foram assumidas para a placa de algodão: densidade de $1500 \mathrm{~kg} / \mathrm{m}^{3}$, calor especifico de $1339,78 \mathrm{~J} /(\mathrm{kg} . \mathrm{K})$ e condutividade térmica de 0,029 W/(m.K), conforme valores usados por Santos (2013). 


\section{FORMULAÇÃO MATEMÁTICA}

Com o objetivo de modelar e simular a fluidodinâmica e a transferência de calor produzida a partir de um injetor de secador Rama com bicos na forma de casca hemi-toroidal, faz-se necessária uma abordagem fenomenológica que acople de forma adequada a turbulência e a transferência de calor no fluido com a transferência de calor no sólido. Para isto, utilizou-se o software COMSOL Multiphysics ${ }^{\circledR}$ versão 4.3 b o qual utiliza o método dos elementos finitos (MEF) para a resolução dos modelos. O método dos elementos finitos (MEF) é um procedimento numérico utilizado para resolução de sistemas de equações diferenciais parciais (EDP) que discretiza o domínio da solução em elementos. Uma solução aproximada para a EDP pode então ser desenvolvida para cada um desses elementos. A solução total é gerada juntando as soluções individuais e, ao mesmo tempo, garantindo a continuidade nas fronteiras entre os elementos (Chapra e Canale, 2008).

Para simular os fenômenos de escoamento e de transferência de calor para este problema, utilizou-se um módulo não isotérmico. Para todas as interfaces de fluxo turbulento não isotérmico, o COMSOL utiliza o tipo de modelo de turbulência RANS padrão e o modelo de transporte de turbulência de Calor padrão Kays-Crawford, que inclui seu próprio número de Prandtl turbulento.

$$
\begin{aligned}
& \nabla \times(\rho u)=0 \\
& \rho(u \cdot \nabla) u=\nabla \cdot\left[-p \mathrm{I}+\left(\mu+\mu_{T}\right)\left(\nabla u+(\nabla u)^{T}\right)-\frac{2}{3}\left(\mu+\mu_{T}\right)(\nabla \times u) \mathrm{I}-\frac{2}{3} \rho k \mathrm{I}\right] \\
& +F \\
& \rho C_{p} u \times \nabla T=\nabla \times\left(k_{t} \nabla T\right)+Q
\end{aligned}
$$

Onde:

$\rho$ é a massa específica $\left(\mathrm{kg} / \mathrm{m}^{3}\right) ; u$ é o vetor velocidade $(\mathrm{m} / \mathrm{s}) ; F$ é a força gravitacional $(\mathrm{N} / \mathrm{m} 3) ; C p$ é o calor específico a pressão constante $(\mathrm{J} /(\mathrm{kg} . \mathrm{K})) ; T$ é a temperatura absoluta $(\mathrm{K}) ; Q$ é a fonte de calor $\left(\mathrm{W} / \mathrm{m}^{3}\right) ; k_{t}$ é o termo de condutividade térmica (W/m.K).

A equação (1) é a equação que representa a conservação de massa. A conservação da quantidade de movimento está descrita conforme a equação (2). Na equação (3) se descreve a conservação da energia, formuladas em termos de temperatura. Para este estudo se usou o modelo de turbulência $\kappa-\varepsilon$ padrão que é o mais utilizado para simulações computacionais, pois apresenta boa convergência e necessita relativamente de pouco recurso computacional.

\subsection{Modelo de Turbulência $\kappa-\varepsilon$}

A turbulência é um fenômeno no qual as partículas do fluido se misturam de forma não linear, tal como é descrito através de diversos modelos de turbulência. Segundo Heuert e Khatchatourian (2007), no modelo de turbulência $\kappa-\varepsilon, \kappa$ é a energia cinética de turbulência (definida como a variação das flutuações em velocidade) e $\varepsilon$ a dissipação do redemoinho de turbulência (a taxa na qual as flutuações de velocidade se dissipam). Os valores de $\kappa-\varepsilon$ vem 
diretamente das equações diferenciais de transporte de energia cinética de turbulência e taxa de dissipação de turbulência, conforme as equações 4 e 5 :

$$
\begin{aligned}
& \rho \frac{\partial k}{\partial t}+\rho u \cdot \nabla k=\nabla \cdot\left(\left(\mu+\frac{\mu_{T}}{\sigma_{k}}\right) \nabla k\right)+P_{k}-\rho \varepsilon \\
& \rho \frac{\partial \varepsilon}{\partial t}+\rho u \cdot \nabla \varepsilon=\nabla \cdot\left(\left(\mu+\frac{\mu_{T}}{\sigma_{s}}\right) \nabla \varepsilon\right)+C_{\varepsilon 1} \frac{\varepsilon}{k} P_{k}-C_{s 2} \rho \frac{\varepsilon^{2}}{k}
\end{aligned}
$$

Onde $u$ é a velocidade, $\rho$ a densidade e $\mu$ a viscosidade do fluido. $C_{s 1}, C_{s 2_{s}} e C_{\mu}$, são constantes e iguais a 1,$44 ; 1,92$ e 0,09 , respectivamente. $\sigma_{k}=1,0$ é número de Prandlt turbulento para $\kappa$ e $\sigma_{\varepsilon}=1,3$ é o número de Prandlt turbulento para $\varepsilon$.

$P_{k}$ é a produção de energia cinética turbulenta devido a forças de flutuabilidade e viscosas, dada por:

$$
P_{k}=\mu_{T}\left(\nabla u:\left(\nabla u+(\nabla u)^{T}\right)-\frac{2}{3}(\nabla \cdot u)^{2}\right)-\frac{2}{3} \rho k \nabla \cdot u
$$

A viscosidade turbulenta $\mu_{T}$ é modelada segundo:

$$
\mu_{T}=\rho C_{\mu} \frac{k^{2}}{\varepsilon}
$$

\section{RESULTADOS E DISCUSSÃO}

Para este trabalho foram estudados dois parâmetros operacionais dentre aqueles de maior influência e que são seguidamente investigados neste tipo de problema, quais sejam: razão entre a distância dos bicos até a superfície a ser aquecida e o diâmetro dos bicos $\left(\mathrm{H} / \mathrm{D}_{\mathrm{b}}\right.$, no presente estudo $D_{b}$ foi mantido constante) e a velocidade de deslocamento da placa (camada) de algodão $\left(\mathrm{V}_{\mathrm{p}}\right)$.

\subsection{Os coeficientes de transferência de calor local e os números de Nusselt}

Os números de Nusselt locais são calculados a partir do gradiente de temperatura sobre a placa plana, conforme descrito na equação 8:

$$
N u_{(x)}=D_{b}(\partial T / \partial y)_{y=0} /\left(T_{a r}-T_{p l a c a}\right)
$$

A partir dos números de Nusselt obtidos com a equação 8 são calculados os coeficientes de transferência de calor locais, conforme a equação 9:

$$
h_{c}=\frac{N u * k_{f}}{D_{b}}
$$

Onde $k_{f}$ é a condutividade térmica do fluido e $D_{b}$ é o diâmetro do bico. 


\subsection{Efeito da razão entre a distância e o diâmetro dos bicos $\left(H / D_{b}\right)$}

Na Figura 3, para a velocidade de $0,4 \mathrm{~m} / \mathrm{s}$ de translação longitudinal da placa de algodão da esquerda para a direita, são apresentados os campos de velocidade e linhas de corrente para diferentes razões entre a altura e o diâmetro dos bicos $\mathrm{H} / \mathrm{D}_{\mathrm{b}}=2 ; 4,6$ e 6 em gráficos $2 \mathrm{D}$ de cortes longitudinais ( 2 e 3 bicos, correspondentes às duas paredes laterais de simetria, tal como explicado no item 2). Pode-se observar que os jatos incidem sobre a superfície com maior velocidade para $\mathrm{H} / \mathrm{D}_{\mathrm{b}}=2$ e 4,6 produzindo remoinhos caraterísticos de escoamentos turbulentos; para $\mathrm{H} / \mathrm{D}_{\mathrm{b}}=6$ os jatos já se apresentam algo deslocados devido ao movimento de translação da placa de algodão.

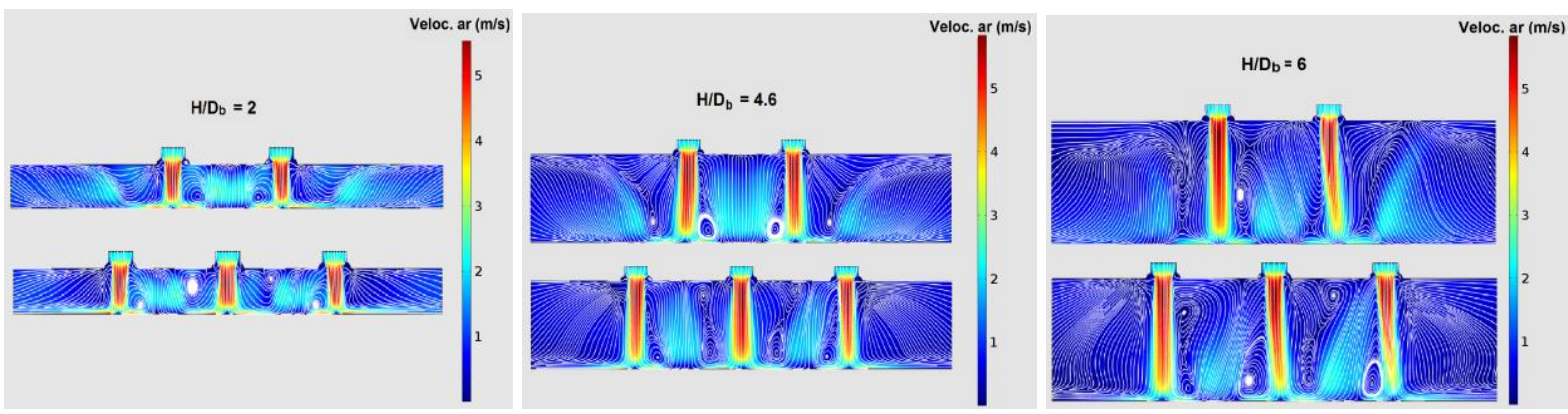

Figura 3- Perfis de velocidade e linhas de corrente para razão entre a altura e o diâmetro dos bicos $H / D_{b}=2 ; 4,6$ e 6 para a velocidade de $0,4 \mathrm{~m} / \mathrm{s}$ da placa de algodão

$\mathrm{Na}$ Figura 4, o conjunto de gráficos permite analisar a distribuição dos coeficientes convectivos locais e do campo de temperaturas sobre a placa de algodão para $H / D_{b}=2 ; 4,6 \mathrm{e}$ 6. Observa-se que os coeficientes convectivos para os três casos são maiores nos pontos de estagnação representados pelos picos vermelhos nas superfícies $3 \mathrm{D}$ e que estes vão diminuindo à medida que se distanciam do ponto de estagnação. O mínimo e o máximo valor de coeficiente convectivo nestas simulações foram 2,08 e $33,45\left(\mathrm{~W} / \mathrm{m}^{2} \mathrm{~K}\right)$, respetivamente. Para a temperatura, observar-se que os valores são muito similares entre as simulações; somente na simulação para $\mathrm{H} / \mathrm{D}_{\mathrm{b}}=2$ obtêm-se valores mais elevados nos pontos de estagnação. Lee e Lee (1999) apresentaram resultados semelhantes obtidos para um jato de choque axissimétrico na região de estagnação; também testaram a dependência do número de Reynolds e de Nusselt no ponto de estagnação para $H / \mathrm{D}_{\mathrm{b}}=2,4,6$, e 10 e observaram que $\mathrm{o}$ menor espaçamento entre o bico e a placa produzia sobre esta maiores gradientes de velocidade e maior turbulência. Lytle e Webb (1994) mostraram que, para um jato não confinado em pequenas distâncias de separação entre bico e placa $\left(0,10<\mathrm{H} / \mathrm{D}_{\mathrm{b}}<6,0\right.$ e 3.600 $<\operatorname{Re}<27.600)$ o número local de Nusselt aumenta em todos os lugares, à medida que a distância de separação é reduzida. 

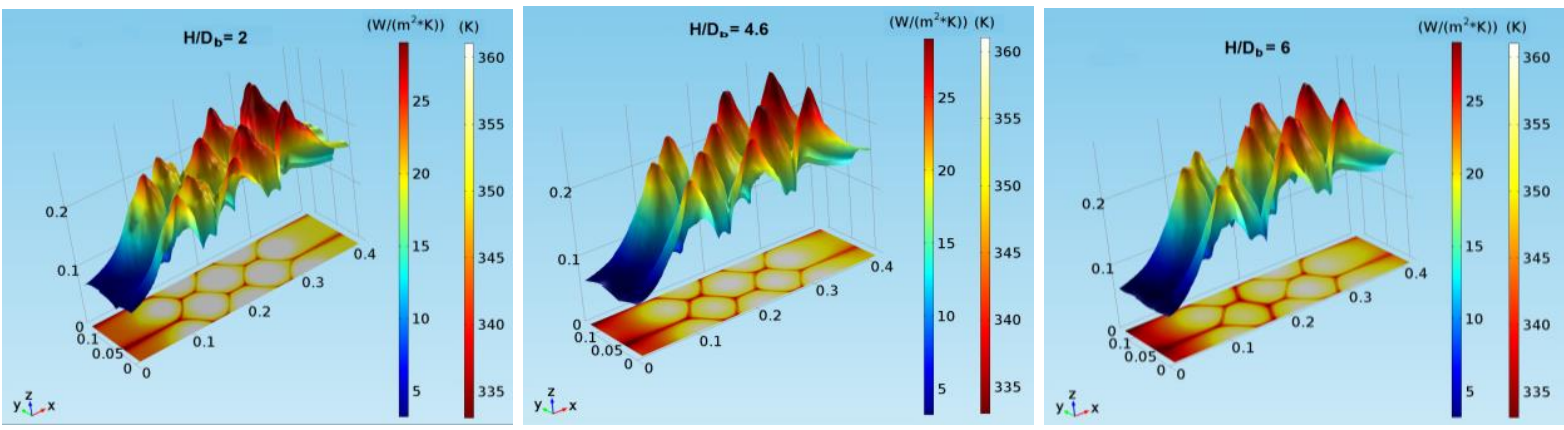

Figura 4- Coeficientes convectivos e temperaturas para razão entre a altura e o diâmetro dos bicos $\mathrm{H} / \mathrm{D}_{\mathrm{b}}=2 ; 4,6$ e 6 .

\subsection{Efeito da velocidade de deslocamento da placa $\left(V_{p}\right)$}

A Figura 5 apresenta os números de Nusselt obtidos para uma velocidade de entrada do ar de $2 \mathrm{~m} / \mathrm{s}$ e uma temperatura de $453,15 \mathrm{~K}$ e uma razão entre a altura dos jatos e o diâmetro dos bicos $H / \mathrm{D}_{\mathrm{b}}=4,6$ para 5 velocidades de deslocamento da placa, $\mathrm{V}_{\mathrm{p}}=0,2 \mathrm{~m} / \mathrm{s} ; 0,4 \mathrm{~m} / \mathrm{s} ; 1$ $\mathrm{m} / \mathrm{s} ; 2 \mathrm{~m} / \mathrm{s}$ e $5 \mathrm{~m} / \mathrm{s}$.

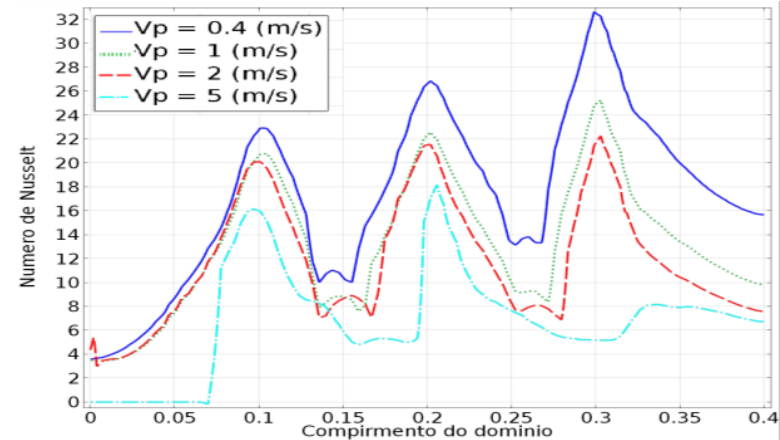

Figura 5- Números de Nusselt para velocidades da placa $V_{p}=0,2 \mathrm{~m} / \mathrm{s} ; 0,4 \mathrm{~m} / \mathrm{s} ; 1 \mathrm{~m} / \mathrm{s} ; 2 \mathrm{~m} / \mathrm{s} \mathrm{e}$ $5 \mathrm{~m} / \mathrm{s}$ a velocidade de $0,4 \mathrm{~m} / \mathrm{s}$ da placa de algodão.

Na Figura 6 são apresentadas as distribuições dos valores dos coeficientes convectivos locais e dos campos de temperatura sobre a superfície de algodão. Os valores mais elevados dos coeficientes convectivos são alcançados quando a velocidade da placa é $0,2 \mathrm{~m} / \mathrm{s}$, atingindo-se o valor máximo de $41,8 \mathrm{~W} / \mathrm{m}^{2} \mathrm{~K}$ no ponto de estagnação; estes valores vão diminuindo à medida que aumenta essa velocidade. Do mesmo modo que para os coeficientes convectivos, as temperaturas mais elevadas se verificam para a velocidade da placa de $0,2 \mathrm{~m} / \mathrm{s}$ $(367,86 \mathrm{~K})$ e diminuem à medida que essa velocidade aumenta. Comportamentos similares foram relatados por Sharif e Banerjee (2008) para um jato de ar sobre uma placa em movimento; nesse trabalho, as velocidades estudadas foram de $0 \mathrm{~m} / \mathrm{s} ; 0,1 \mathrm{~m} / \mathrm{s} ; 0,5 \mathrm{~m} / \mathrm{s} ; 1 \mathrm{~m} / \mathrm{s}$ e $2 \mathrm{~m} / \mathrm{s}$ para $(5.000<\mathrm{Re}<20.000$ e $\mathrm{H} / \mathrm{D}=6$ e 8). Os autores concluíram que, quando a velocidade da placa é baixa, o efeito do jato controla o comportamento da transferência de calor, e que, à medida que a velocidade da placa aumenta, o cisalhamento devido ao movimento da placa domina gradualmente os campos térmicos, o que faz com que os padrões de fluxo e isotermas sejam distorcidos e desviados no sentido do movimento da placa. 


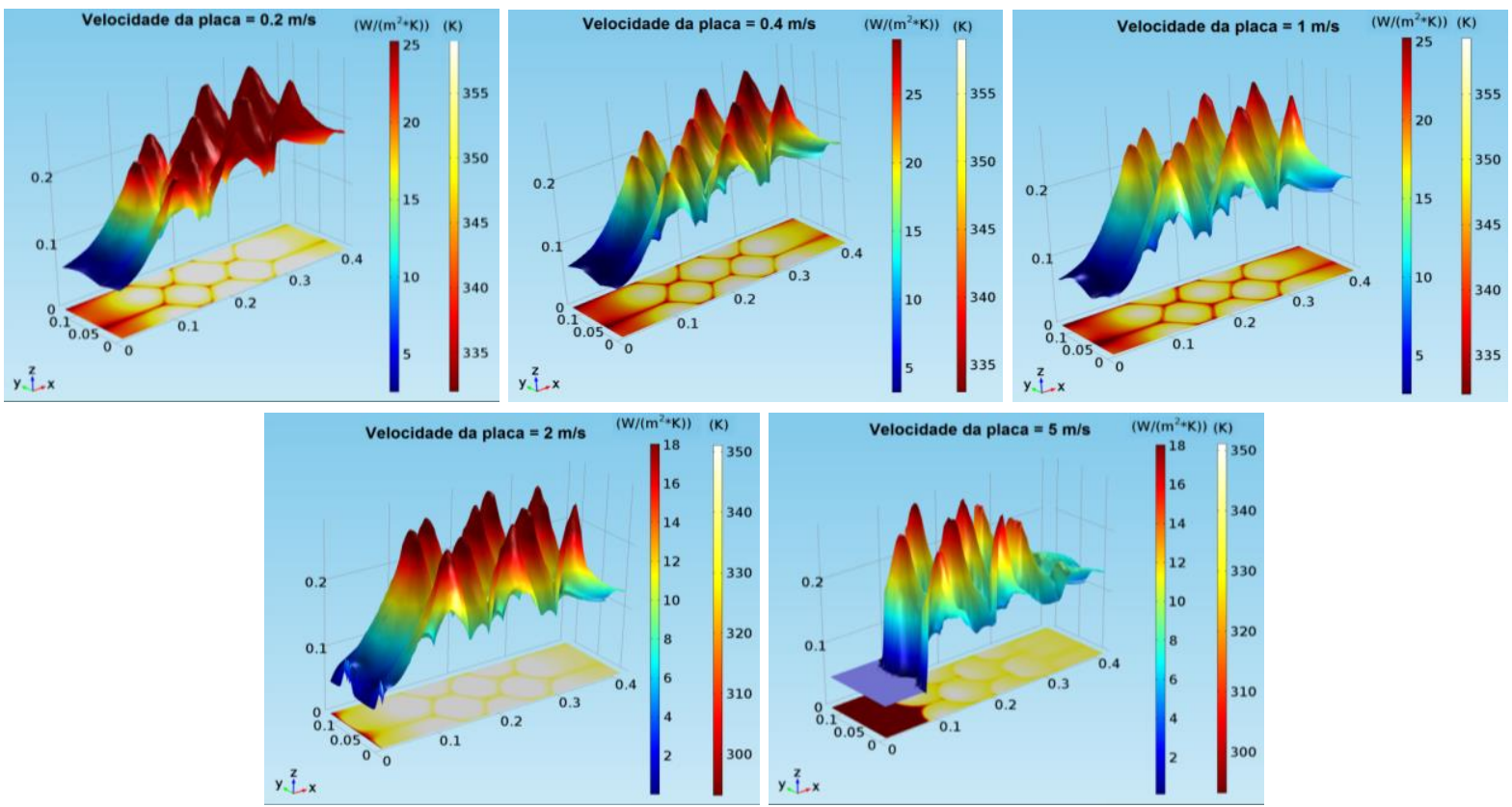

Figura 6- Coeficientes convectivos e temperaturas para velocidades da placa $V_{p}=0,2 \mathrm{~m} / \mathrm{s} ; 0,4$ $\mathrm{m} / \mathrm{s} ; 1 \mathrm{~m} / \mathrm{s} ; 2 \mathrm{~m} / \mathrm{s}$ e $5 \mathrm{~m} / \mathrm{s}$.

\section{CONCLUSÕES}

Analisou-se as características de transferência de calor para uma configuração de 5 bicos com borda hemi-toroidal direcionados perpendicularmente sobre uma superfície de algodão. Foram testadas a razão entre a altura e o diâmetro dos bicos $\left(\mathrm{H} / \mathrm{D}_{\mathrm{b}}\right)$ e a velocidade da placa $\left(\mathrm{V}_{\mathrm{p}}\right)$. As tendências observadas foram similares àquelas relatadas na literatura para outros tipos de jatos. Os maiores coeficientes são alcançados na razão $H / D_{b}=2$ e diminuem gradualmente para $H / D_{b}=$ 4,6 e 6. Isto é devido aos maiores gradientes de velocidade e maior turbulência quando a altura do bico em relação à superfície de choque é menor. Os valores mais elevados dos coeficientes convectivos são alcançados quando a velocidade da placa é de $0,2 \mathrm{~m} / \mathrm{s}$, atingindo $41,8 \mathrm{~W} / \mathrm{m}^{2} \mathrm{~K}$ no ponto de estagnação; à medida que essa velocidade aumenta, diminuem os coeficientes convectivos devido ao cisalhamento e desvio do jato do ar no sentido do movimento da placa. A razão entre a altura e o diâmetro dos bicos, bem como a velocidade de deslocamento da placa demostraram ter grande influência sobre a transferência de calor e, portanto, são parâmetros operacionais que, juntamente com as propriedades do material a secar, devem ser considerados para a otimização da eficiência de secadores Rama.

\section{REFERÊNCIAS}

CHAPRA, S. C.; CANALE, R.P. Métodos numéricos para engenharia. $5^{\text {a }}$ ed. McGraw Hill, 2008.

CHATTOPADHYAY, H.; SAHA, S.K. Turbulent heat transfer from a slot jet impinging on a moving plate. Int. J. of Heat and Fluid Flow. v.24, p. 685-697, 2003.

COLUCCI, D.W.; VISKANTA, R. Effect of Nozzle Geometry on Local Convective Heat Transfer to a Confined Impinging Air Jet. Exp. Ther. and Fluid Sci. v. 13, p.71-80, 1996. 
FERRAZ, A. D et al. Eficiência na indústria têxtil: oportunidades para o gás natural. Rio Oil \& Gas Expo and Conference 2010. Instituto Brasileiro de Petróleo, Gás e Biocombustíveis - IBP, Rio de Janeiro, 2010.

GULATI, P.; KATTI, V.; PRABHU, S.V. Influence of the shape of the nozzle on local heat transfer distribution between smooth flat surface and impinging air jet. Int. J. of Ther. Scie. v. 48, p. 602-617, 2009.

HEUERT, J.; KHATCHATOURIAN, O. Interação entre Jatos Transversais e Fluxo Principal: Simulação e Análise Comparativa de Modelos de Turbulência. Congresso Nacional de Matemática Aplicada e Computacional, Florianópolis, 3 a 6 setembro 2007.

LEE, J.; LEE S.J. The efect of nozzle configuration on stagnation región heat transfer enhancement of axisymmetric jet impingement. Int. J. Heat Mass Trans. v. 43, p. 3497$3509,2000$.

LYTLE, D.; WEBB, B. W. Air Jet Impingement Heat Transfer at Low Nozzle-Plate Spacings. Int. J. Heat Mass Trans. v. 37, p.1687-1697, 1994.

SANTOS, R. Estudo da Secagem e do consumo de Gás natural em uma Rama têxtil de aquecimento direto. Dissertação (Mestrado em Engenharia Química), Universidade Federal de Santa Catarina, Florianópolis, p. 82, 2013.

SHARIF, M.A.R.; BANERJEE, A. Numerical analysis of heat transfer due to confined slotjet impingement on a moving plate. Applied Ther. Eng. v. 29, p. 532-540, 2009. 